\title{
Cascos fríos para la prevención de alopecia inducida por quimioterápicos
}

\author{
Cold caps for the prevention of chemotherapy-induced alopecia
}

Candela Agustina Loza ${ }^{\text {(D) }}$, Milagros Medina ${ }^{a}$ (D)

\begin{abstract}
Resumen
Uno de los efectos adversos que generan mayor impacto psicosocial en los pacientes que se someten a quimioterapia es la pérdida de cabello. El uso de los cascos fríos podría representar una alternativa para prevenir la alopecia. Estos dispositivos producen vasoconstricción en los vasos sanguíneos del cuero cabelludo provocando que los quimioterápicos lleguen en menores concentraciones a los folículos pilosos, y por consiguiente, evitando que los citotóxicos alcancen el metabolismo de las células foliculares. Se propone que este proceso permita prevenir la alopecia, aunque existe una preocupación que es la posibilidad de metástasis en cuero cabelludo si la quimioterapia no llega a este tejido. Partiendo de la consulta de una paciente con diagnóstico reciente de cáncer de mama, surge el interrogante si en pacientes en tratamiento con quimioterapia el uso de cascos fríos en comparación con su no utilización, disminuye la caída del cabello y si aumenta el riesgo de metástasis en cuero cabelludo. Luego de realizar una búsqueda, se analizaron los resultados de tres artículos pertinentes. Se concluye que el uso de cascos fríos en pacientes que reciben quimioterapia representaría una opción válida para prevenir la alopecia sin indicios de problemas de seguridad y se realiza una reseña acerca de la disponibilidad de los cascos fríos en Argentina.
\end{abstract}

\begin{abstract}
One of the adverse effects that generate greater psychosocial impact in patients undergoing chemotherapy is hair loss. The use of cold caps could represent an alternative to prevent alopecia. These devices produce vasoconstriction in the blood vessels of the scalp, causing chemotherapeutic agents to reach the hair follicles in lower concentrations, and therefore preventing toxins from being metabolized by the follicular cells. Thus, alopecia could be prevented but, there is a concern that is the possibility of metastasis to the scalp if the therapy does not reach this tissue. Based on a consultation of a patient recently diagnosed with breast cancer, the question arises whether in patients undergoing chemotherapy the use of cold caps compared to their non-use reduces hair loss and whether it increases the risk of metastasis to the scalp. After performing a search, the results of three relevant articles were analyzed. It is concluded that the use of cold helmets in patients receiving chemotherapy would represent a valid option to prevent alopecia without indications of safety problems. Finally, a review is made about the availability of scalp-cooling devices in Argentina.
\end{abstract}

Palabras clave: Enfriamiento, Alopecia, Quimioterapia, Neoplasias de la Mama, Neoplasias. Keywords: Cooling, Alopecia, Drug Therapy, Breast Neoplasms, Neoplasms.

Loza CA, Medina M.. Cascos fríos para la prevención de alopecia inducida por quimioterápicos. Evid Actual Pract Ambul. 2020;23(4):e002101.

\section{Escenario clínico}

Se presenta a la consulta una mujer de 63 años de edad con diagnóstico reciente de cáncer de mama, por el cual comenzará a recibir tratamiento quimioterápico. Refiere sentirse angustiada por la inminente alopecia, por lo que pregunta al médico de familia su opinión acerca de la posibilidad de utilizar cascos fríos para prevenir este problema durante el periodo en el que reciba la quimioterapia.

\section{Pregunta que generó el caso}

En pacientes con cáncer en tratamiento con quimioterapia, ¿el uso del casco frío en comparación con la no utilización de este dispositivo, disminuye la caída del cabello y/o aumenta el riesgo de metástasis en cuero cabelludo?

\section{Estrategia de búsqueda}

Se realizaron búsquedas en PubMed empleando las combinaciones de términos '(Cold OR cooling OR cool) AND (cap OR scalp) AND (Alopecia OR hair loss)' y '(Cold OR cooling OR cool) AND (cap OR scalp) AND (scalp metastases)'. Se aplicaron filtros para priorizar la recuperación de revisiones sistemáticas y ensayos clínicos.

\section{Algunos datos sobre los cascos fríos}

Hoy en día, el cáncer es una enfermedad de alta incidencia que no sólo repercute en la esfera física sino también tiene grandes implicancias psicológicas. Los pacientes tienden a tomar el rol social de enfermo, conviven con sentimientos de angustia, y los cambios de apariencia por los tratamientos a los que se someten no son de gran ayuda.

Una de las principales terapias es la quimioterapia en sus diversas variedades, según el tipo de cáncer y el estadío de la enfermedad. La quimioterapia no suele realizarse con una única droga constituye un grupo heterogéneo de intervenciones con diversa toxicidad, esquemas y modalidades de tratamiento. La quimioterapia citotóxica se suele asociar a diferentes toxicidades o efectos adversos, pero la alopecia es uno de los signos más visibles. Aparece entre el primer y segundo ciclo de tratamiento, y genera un gran impacto psicosocial en la persona enferma. La quimioterapia citotóxica no discrimina entre células sanas y cancerígenas, daña a los queratinocitos, las células que conforman a los folículos pilosos, causando esta manifestación tan angustiante para los pacientes ${ }^{1}$.

Los cascos fríos, implementados hace más de 50 años, representan una de las alternativas más utilizadas para combatir la alopecia. Los mismos se usan antes, durante y después de cada

\footnotetext{
a Carrera de Medicina, Instituto Universitario Hospital Italiano de Buenos Aires. candela.loza@hospitalitaliano.org.ar, milagros.medina@hospitalitaliano.org.ar
} 
ciclo de quimioterapia, a una temperatura específica (entre $-18^{\circ} \mathrm{C}$ y $-22^{\circ} \mathrm{C}$ ). El enfriamiento capilar puede realizarse de dos formas; una más sencilla, consiste en cascos congelados (Figura 1) que deben cambiarse con regularidad, lo que resulta en fluctuaciones de la temperatura. El segundo mecanismo consiste en el uso de dispositivos más sofisticados (Figura 2), los cuales contienen un casco personalizado y una unidad que controla el enfriamiento. En este caso, el frío circula por el casco manteniendo la temperatura predefinida de forma constante, evitando así las fluctuaciones de temperatura. La eficacia de ambas estrategias puede verse influenciada por diversos factores, incluyendo las características de las personas, el tiempo de uso y la temperatura del método utilizado para el enfriamiento del cuero cabelludo, y el tipo de agentes usados en la quimioterapia, con sus respectivas dosis y regímenes ${ }^{1}$.

Figura 1. Casco frío confeccionado con gel refrigerante congelado (ilustración de las autoras)

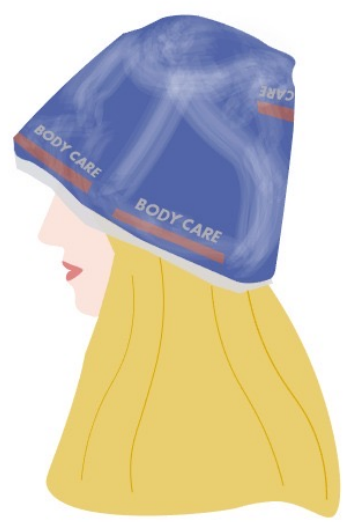

Figura 2. Casco frío comercial con sistema electrónico de control de temperatura (ilustración de las autoras)

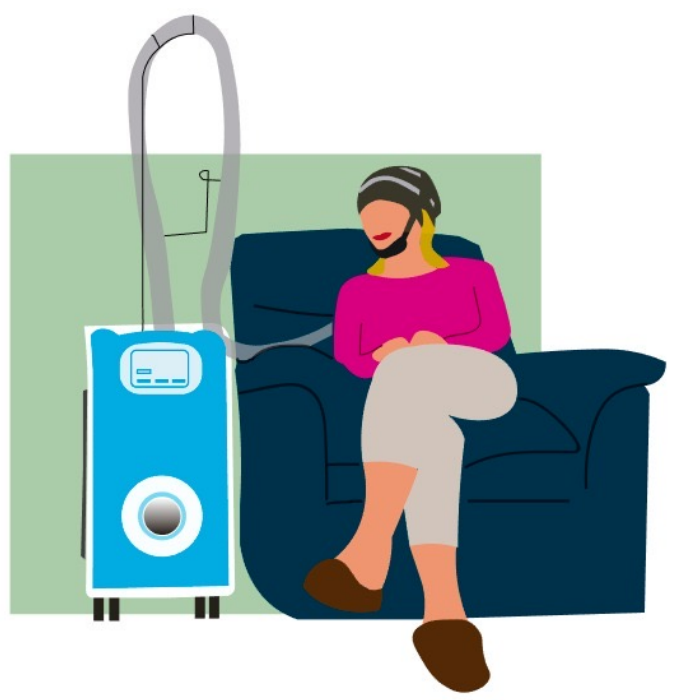

Los cascos fríos producen vasoconstricción en los vasos sanguíneos del cuero cabelludo, lo que permite que la quimioterapia llegue en menores concentraciones a los folículos pilosos, y por consiguiente, evita sus efectos tóxicos en las células foliculares ${ }^{1}$. A partir de este mecanismo se podría llegar a prevenir la caída de cabello, pero existe otra preocupación: la posibilidad de metástasis en cuero cabelludo si la quimioterapia no llega a ese tejido.

\section{Resumen de evidencia}

Se seleccionaron tres artículos que parecían los más relevantes para responder a las preguntas planteadas: dos revisioness sistemática de ensayos clínicos aleatorizados (ECA $)^{2,3}$ y un ECA publicado con fecha posterior, no incluido en las revisiones mencionadas ${ }^{4}$.

\section{Rugo HS, et al. Scalp Hypothermia for Preventing Alopecia} During Chemotherapy. A Systematic Review and Meta-Analysis of Randomized Controlled Trials. Clin Breast Cancer. 2018 Feb;18(1):19-28

El objetivo principal de esta revisión sistemática ${ }^{2}$ fue determinar si la utilización del enfriamiento del cuero cabelludo en comparación a su no utilización, reducía significativamente la alopecia cuando se emplea junto a regímenes de quimioterapia para el cáncer.

Para la realización de esta revisión sistemática y meta-análisis de ECA y controlados, se incluyeron artículos encontrados en búsquedas de bases electrónicas de datos hasta febrero de 2017 , sin restricciones por idioma. Los datos se recolectaron y evaluaron mediante el uso de un formulario de recolección de datos, y la evaluación del riesgo de sesgo se realizó siguiendo los métodos estándar de Cochrane. Se utilizó la metodología GRADE para valorar la calidad de la evidencia.

Se encontraron 221 artículos, de los cuales se incluyeron 10 estudios con 654 pacientes, la mayoría con diagnóstico de cáncer de mama (432 personas, $66 \%$ ), en tratamiento con antraciclinas. Los resultados observados fueron que el uso de cascos fríos redujo en $46 \%$ el riesgo relativo de desarrollar alopecia (Riesgo Relativo [RR] 0,54; Intervalo de Confianza [IC] del 95\% 0,46 a 0,$63 ; I^{2} 11 \%$, cinco estudios, 295 participantes, calidad de la evidencia moderada). No se encontró evidencia de que el empleo de estos dispositivos aumente la incidencia de metástasis en cuero cabelludo (desenlace no reportado en los estudios primarios).

Los autores concluyeron que esta revisión sistemática apoya el uso del enfriamiento del cuero cabelludo para prevenir la alopecia en pacientes con tumores sólidos que realicen tratamiento quimioterápico. Sin embargo, una de las limitaciones que presenta el trabajo es la reducida cantidad de ensayos aleatorizados disponibles, con un número bajo de pacientes, y el alto riesgo de sesgo de los estudios incluidos, en los cuales no hubo cegamiento.

Rugo HS, et al. Scalp cooling with adjuvant/neoadjuvant chemotherapy for breast cancer and the risk of scalp metastases: systematic review and meta-analysis.Breast Cancer Res Treat. 2017; 163(2): 199-205

El objetivo de esta revisión sistemática y meta-análisis de estudios longitudinales ${ }^{3}$ fue evaluar el riesgo de desarrollar metástasis en el cuero cabelludo en las personas con cáncer de mama que emplearon dispositivos de enfriamiento de este tejido en comparación con quienes no los utilizaron, para la preservación del cabello durante la quimioterapia.

Se realizaron búsquedas electrónicas, en revistas específicas, y búsquedas manuales en las listas de referencias de los artículos identificados. Los pacientes fueron apareados sobre la base de la enfermedad, el tratamiento, la ausencia de metástasis y el sexo.

Se identificaron un total de 24 artículos de texto completo, de los cuales 10 cuantificaron la incidencia de metástasis en el cuero 
cabelludo. Para pacientes con enfriamiento del cuero cabelludo, se evaluaron 1.959 pacientes con una media de seguimiento de 43,1 meses. Para los pacientes sin enfriamiento del cuero cabelludo, se evaluaron 1.238 pacientes con una media de seguimiento de 87,4 meses. La tasa de incidencia de metástasis del cuero cabelludo del grupo que recibió enfriamiento frente a quienes no recibieron enfriamiento fue $0,61 \%$ (IC $95 \% 0,32$ a $1,1 \%$ ) vs. $0,41 \%$ (IC $95 \% 0,13$ a $0,94 \%$ ); $P=0,43$.

Los autores concluyeron que la incidencia de metástasis fue baja independientemente del uso de estrategias de enfriamiento del cuero cabelludo, y que el análisis de los datos no sugieren que el uso de esta tecnología para prevenir la alopecia por la quimioterapia incremente la incidencia de metástasis en esta localización.

\section{Smetanay K, et al. COOLHAIR: a prospective randomized trial to investigate the efficacy and tolerability of scalp cooling in patients undergoing (neo)adjuvant chemotherapy for early breast cancer. Breast Cancer Res Treat. 2019 Jan;173(1):135-143.}

En este ECA ${ }^{4}$ abierto con 79 pacientes realizado en Alemania entre 2014 y 2016, se incluyeron pacientes mayores de 18 años, con diagnóstido de cáncer de mama estadio en estadíos I a III, con poca afectación de su estado general y quimioterapia neoadyuvante planificada con antraciclinas o taxanos, que fueron aleatorizados a recibir enfriamiento del cuero cabelludo u observación. El desenlace primario fue la tasa de alopecia autorreportada de acuerdo a una versión modificada de la escala Dean. La preservación del cabello fue definida como la pérdida de cabello menor o igual al $50 \%$ (menor o igual al grado 2 de la escala). Los desenlaces secundarios fueron la tasa de alopecia determinada por los profesionales médicos, la tasa de uso de pelucas o turbantes, la tolerabilidad y la calidad de vida.

Se aleatorizaron 41 pacientes a la rama de cascos fríos y 38 pacientes a no usar cascos. La preservación del cabello se observó en el $39,3 \%$ de los pacientes que utilizaron cascos fríos versus $0 \%$ en el grupo de pacientes que no los emplearon $(p<0,001)$. El uso de pelucas/turbantes fue significativamente menos frecuente en el grupo que utilizó cascos fríos $(40,7 \%$ vs $95,5 \%, p<0,001)$. No hubo diferencias significativas en la calidad de vida. No se observaron diferencias en la eficacia entre los regímenes basados en antraciclinas y los no basados en este grupo de drogas.

Dentro de los eventos adversos se observaron escalofríos graves en $63,1 \%$ de los pacientes, mientras que el $18,4 \%$ sufrió dolores de cabeza severos. Dos pacientes interrumpieron el enfriamiento del cuero cabelludo debido a eventos adversos relacionados con el dispositivo.

Los autores el ensayo concluyen que este estudio provee evidencia de que el uso de cascos fríos previene la alopecia en un número significativo de pacientes, y que esta opción debería estar disponible para las personas que deban realizar quimioterapia neoadyuvante para el tratamiento del cáncer de mama en estadios iniciales. Sin embargo, dentro de sus limitaciones, se destacan que el estudio contaba con un número pequeño de individuos con los que se armaron los grupos de estudio, y el alto riesgo de sesgo por pérdida de datos, ya que las tasas de abandono del ensayo clínico fueron de $31,7 \%$ y $34,2 \%$ en el grupo de pacientes que utilizó cascos fríos y en el grupo que no lo hizo, respectivamente. Las principales razones para el abandono de los pacientes fueron la pérdida de cabello, la aparición de efectos adversos dentro del grupo que empleó los cascos fríos y la decepción de ser asignados al grupo control, por lo que es necesario además considerar como fuente de sesgo a la falta de cegamiento de la intervención evaluada.

\section{Conclusiones y recomendaciones}

A partir de la evidencia analizada, consideramos que el uso de cascos fríos en pacientes que reciben quimioterapia podría representar una opción válida para prevenir la alopecia inducida por su citotoxicidad para el cuero cabelludo. No se asociaría a eventos adversos graves como metástasis, pero podrían producir escalofríos importantes, cefalea, e incluso lesiones térmicas de la piel ${ }^{5}$. Cabe destacar que, dado que la mayoría de los participantes incluidos en los estudios evaluados tenían diagnóstico de cáncer de mama, el cual tiene baja incidencia de metástasis en el cuero cabelludo, estas conclusiones podrían no ser extrapolables a otros tipos de cánceres sólidos u oncohematológicos.

Basándose en parte en los resultados de las investigaciones resumidas en este artículo, la actualización más reciente de la guía de práctica clínica para el manejo del cáncer de mama de la National Comprehensive Cancer Network (NCCN, una alianza sin fines de lucro de centros oncológicos de los EE.UU.), incluyó entre sus recomendaciones para el tratamiento de las personas con cáncer de mama metastásico que el uso de la tecnología de enfriamiento del cuero cabelludo sea considerado para reducir la incidencia de alopecia inducida por la quimioterapia ${ }^{6}$.

En la actualidad, tanto los cascos fríos confeccionados con geles refrigerantes como los que utilizan dispositivos electrónicos más complejos se encuentran disponibles en Argentina desde 2017, con autorización de la Agencia Nacional de Medicamentos, Alimentos y Tecnología Médica ${ }^{7}$, con costos variables. Los más sofisticados son comercializados por empresas con fines de lucro que proporcionan tanto los cascos como la máquina a la cual se conectan, la que puede ser adquirida por cada institución, hasta técnicos especialistas que acompañan al paciente y programan la totalidad del tratamiento en cada sesión, brindando de esta forma un servicio tercerizado. El acceso a dichas prestaciones depende del pago de bolsillo del paciente ya que no se contempla como prestación cubierta por prepagas y obras sociales (excepto en la ciudad de Viedma, Río Negro). El valor del servicio puede oscilar entre los 3 mil y 10 mil pesos argentinos por aplicación, dependiendo la disponibilidad de soporte técnico.

Como alternativa, los cascos fríos confeccionados con sachets de geles refrigerantes pueden estar al alcance de una mayor proporción de pacientes debido a su fácil acceso y bajo costo (alrededor de 600 pesos argentinos por casco). Estos se construyen con envases de un polímero artificial que funciona como substituto práctico del hielo, que se moldean según la cabeza de cada paciente. Una vez tomada la forma, se depositan en el freezer para su congelación. Los pacientes acuden a la sesión de quimioterapia con una heladera de mano que mantiene la temperatura de los diez cascos congelados necesarios para cada sesión de quimioterapia. El casco se coloca una hora antes del inicio de la sesión con el cabello mojado, luego se reemplaza por otro cada media hora y, una vez finalizada la sesión, debe mantenerse colocado durante una hora más.

En Argentina existe una red de contacto entre personas que desean obtener este tratamiento, principalmente a través de las redes sociales. La organización pionera en el empleo de cascos fríos en el país, "Quimio con Pelo", tiene a su disposición los cascos y los brinda de forma gratuita a las personas que los requieran $^{8}$. El principal requisito impuesto por la organización para la utilización de los cascos constituye la autorización por parte del equipo médico. En comunicación personal con miembros de esta organización sin fines de lucro informan que han utilizado este servicio pacientes con cáncer de mama, ovario, útero, pulmón y linfoma, aunque no se conoce en profundidad acerca de la indicación en estos últimos y, de hecho, la Administración de Drogas y Alimentos de los EE.UU. (FDA, por sus iniciales en inglés) aprobó el empleo de cascos fríos exclusivamente para pacientes con tumores sólidos ${ }^{9}$. 


\section{Referencias}

1. de Barros-Silva G, Ciccolini K, Donati A, et al. Scalp cooling to prevent chemotherapy-induced alopecia. An Bras Dermatol. 2020;95(5):631-637. Available from: 10.1016/j.abd.2020.03.005.

2. Rugo HS, Voigt J. Scalp Hypothermia for Preventing Alopecia During Chemotherapy. A Systematic Review and Meta-Analysis of Randomized Controlled Trials. Clinical Breast Cancer. 2018;18(1):19-28. Available from: 10.1016/j.clbc.2017.07.012.

3. Rugo HS, Melin SA, Voigt J. Scalp cooling with adjuvant/neoadjuvant chemotherapy for breast cancer and the risk of scalp metastases: systematic review and meta-analysis. Breast Cancer Research and Treatment. 2017;163(2):199-205. Available from: 10.1007/s10549-017-4185-9.

4. Smetanay K, Junio P, Feißt M, et al. COOLHAIR: a prospective randomized trial to investigate the efficacy and tolerability of scalp cooling in patients undergoing (neo)adjuvant chemotherapy for early breast cancer. Breast Cancer Research and Treatment. 2019;173(1):135-143. Available from: 10.1007/s10549-018-4983-8.

5. Hagigeorges D, Burns LJ, Isakoff SJ, et al. Thermal Injury in a Patient Using a Scalp Cooling System to Prevent Chemotherapy-Induced Alopecia. JCO Oncol Pract. 2020;16(8):522-524. Available from: 10.1200/OP.20.00021.

6. Gradishar WJ, Anderson BO, Abraham J, et al. Breast Cancer, Version 3.2020, NCCN Clinical Practice Guidelines in Oncology. J Natl Compr Canc Netw. 2020;18(4):452-478. Available from: 10.6004/jnccn.2020.0016.

7. ANMAT, Argentina. Disposición 8142-17; 2017. Available from: http://www.anmat.gov.ar/boletin_anmat/julio_2017/Dispo_8142-17.pdf.

8. Cascos fríos para finalizar la "Quimio con pelo"; 2019. Available from: https://www.diarioelzondasj.com.ar/cascos-frios-para-finalizar-la-quimio-conpelo/.

9. FDA clears expanded use of cooling cap to reduce hair loss during chemotherapy; 2017. Available from: https://www.fda.gov/news-events/pressannouncements/fda-clears-expanded-use-cooling-cap-reduce-hair-loss-during-chemotherapy. 